\title{
Philip's Semi-Analytical Solution on Water Infiltration into Unsaturated Soil based on Van Genuchten Equation
}

\author{
Eng Giap Goh ${ }^{1}$
}

\begin{abstract}
Water flow equation in unsaturated soil is based on Richards' equation. The equation could be solved using semianaly tical solution. Philip first coined the solution in 1957. Due to the mathematical complexity employed by Philip, its mathematical solution at that time remained inaccessible to many researchers. The solution was first made comprehensible by Kirkham and Powers in 1972 by providing details account by describing all steps used in the mathematical derivation. The solution has significant implication that it could be adapted to model diffusion process of other applications like contaminant transport or heat flow, and it can be used to validate numerical model that use to govern the diffusive process. When combined with global sensitivity analysis and inverse method, it could be used to study uncertainty of model input and also to estimate input parameter, respectively. In this study, the solution given by Kirkham and Powers was programmed to simulate water flow in unsaturated soil. The currently available data, in the literature, for coded model is to compare with Haverkamp and his co-worker's dataset. The dataset has a significant drawback because it was based on a different equation than the one commonly applied by soil physics community, i.e. van Genuchten constitutive functions. A new dataset based on van Genuchten equation was generated for sandy loam at 6, 12 and 30 mins, and it has been validated by numerical solution. The new dataset will be a useful reference for modeler in model validation.
\end{abstract}

Keywords - sandy loam, van Genuchten constitutive functions, Richards' equation, water flow in unsaturated soil.

\section{INTRODUCTION}

$\mathrm{P}$ hilip in 1957 [1] solution is applied on the diffusion of onedimensional flow problem. The flow path can be directed upward, downward, or horizontal. Various phenomena, such as heat, moisture, molecules and solute flow, that governed by diffusion equation can be solved by Philip's semi-analytical

Eng Giap Goh1 is with the School of Ocean Engineering, Universiti Malaysia Terengganu, 21030 Kuala Terengganu, Terengganu, Malaysia (corresponding author's phone: +6019-9383968; e-mail: sunnygoh@gmail.com).

Firstname SurnameAuthor ${ }^{3}$ is with the Electrical Engineering Department, University of Colorado, Boulder, CO 80309 USA, on leave from the National Research Institute for Metals, Tsukuba, Japan . solution. From now on, Philip's semi-analytical solution is written as PSA. In solving Richards' equation [2] that is used to govern variable water saturation media, Philip first solved for horizontal flow (1) using Boltzmann transformation (3) that is then extended to solve for vertical flow (2) to include the effect of gravitational force.

$$
\begin{gathered}
\frac{\partial \theta_{L}}{\partial t}=\frac{\partial}{\partial x}\left(D \frac{\partial \theta_{L}}{\partial x}\right) \\
\frac{\partial \theta_{L}}{\partial t}=\frac{\partial}{\partial x}\left(D \frac{\partial \theta_{L}}{\partial x}\right)+\frac{\partial K}{\partial x} \\
\lambda\left(\theta_{L}\right)=x t^{-\frac{1}{2}}
\end{gathered}
$$

PSA method is known to be free from specific dependence between diffusivity ( ) and volumetric water content ( ). The independence also implies that the relations given by and can be governed by any constitutive functions, i.e. van Genuchten [3], Haverkamp [4], and so forth. The is matric pressure head and is the hydraulic conductivity at variably water saturation in soil. Water infiltration data generated by PSA method has significant important because modeler could use the data to validate their numerical simulation results before applying to other cases, e.g. Noborio [5]. However, the widely made available water infiltration data in the literature is from Haverkamp [4] who have solved water infiltration problem on Yolo light clay and sand media, but the constitutive functions used are different from the widely used van Genuchten constitutive functions for matric pressure head and hydraulic conductivity. In the current study, PSA method is applied to solve Richards' equation and the relation between diffusivity ( ) and volumetric water content ( ) used is estimated from van Genuchten constitutive functions. Sandy loam is used as the water infiltration media.

\section{MATERIALS AND METHODS}

The following sub-sections summarized the semi-analytical solution used to solve Richards' equation in unsaturated soil. Philip invented the solution in 1957, but the mathematical solution remained unknown to many until Kirkham and Powers 
[6] uncover the mathematical steps in significant details. For detail description of the solution for horizontal and vertical flow, refer to Kirkham and Powers [6]. The mathematical equation and solution described in sub-sections $\mathrm{A}$ and $\mathrm{B}$ were merely summarized from Kirkham and Powers [6], except in Table 1 some variables were newly derived.

\section{A. Horizontal Flow Solution}

In solving horizontal flow, Boltzmann transformation is used to remove spatial variable ( ) and time ( ) from the partial derivative of (1). The Boltzmann-transformed partial differential equation is then integrated from initial background volumetric water content value ( ) to variably volumetric water content, as follows:

$$
\int_{\theta_{n}}^{\theta_{x}} \lambda d \theta=-2 D\left(\theta_{x}\right)\left(\frac{d \theta_{L}}{d \lambda}\right)_{\theta_{x}}
$$

By using histogram rectangles, where as $\mathrm{y}$-axis and as $\mathrm{x}$ axis, a finite difference solution of (4) is established to represent each rectangle of the histogram. Also, two formulae to represent area under the curve by removing the successive rectangles are developed separately and then combined to form a single formula that can be used to govern successive reduction of area under the curve by successive reduction of rectangles. Finite difference solution of (4) and the combined equation, together they formed sequence of formulae to compute . Hence, it generates curve of provided that if and only the area ( ) under the curve and relation are known. Moreover, the estimation of area may be wrong and it requires a second method for comparison. This is done by comparison the area value at rectangle of histogram. The resulted value () given by the subtraction between the first ( ) and second ( ) method as would be used to update the area value and then subject to the same sequence of formulae to compute . until the value, as described, to be insignificantly small. Finally, it generates curve. The curve can be utilized by first rearranging (3) into (5). The volumetric water content values corresponding to the rectangles of the histogram are located between initial background volumetric water content value ( ) and imposed boundary volumetric water content ( ), they give direct relation to the values. By specifying the time of infiltration and the volumetric water content values corresponding to the rectangles of the histogram, the spatial variable ( ) can be calculated by (5).

$$
x=\lambda\left(\theta_{L}\right) t^{\frac{1}{2}}
$$

\section{B. Vertical Flow Solution}

Vertical flow governing equation (2) includes the effect of gravitational force that is in addition to horizontal flow (1). PSA solution does not solve vertical flow equation directly, but it solves horizontal flow equation by taking into consideration layers of error resulted by the difference between vertical and horizontal flow equations. For easy understanding and a clear distinction, horizontal flow equation is rewritten as follows:

$$
\frac{\partial \theta_{L}}{\partial t}=\frac{\partial}{\partial x^{\prime}}\left(D \frac{\partial \theta_{L}}{\partial x^{\prime}}\right)
$$

Horizontal flow (6) and vertical flow (2) equations that have volumetric water content ( ) as dependent variable on the left side of the equations. It must first be transformed to and as dependent variables, corresponding to (7) and (8) as follows:

$$
\begin{gathered}
\frac{\partial x^{\prime}}{\partial t}=-\frac{\partial}{\partial \theta_{L}}\left(D \frac{\partial \theta_{L}}{\partial x^{\prime}}\right) \\
\frac{\partial x}{\partial t}=-\frac{\partial}{\partial \theta_{L}}\left(D \frac{\partial \theta_{L}}{\partial x}\right)+\frac{\partial K}{\partial \theta_{L}}
\end{gathered}
$$

By subtracting (7) from (8), it results in the following:

$$
\frac{\partial y}{\partial t}=\frac{\partial}{\partial \theta_{L}}\left[D\left(\frac{\partial \theta_{L}}{\partial x^{\prime}}\right)\left(\frac{\partial y}{\partial x}\right)\right]+\frac{\partial K}{\partial \theta_{L}}
$$

(9) is then approximated using $\frac{\partial y}{\partial x}=\frac{\partial y}{\partial x^{\prime}}$ and $y=y^{\prime}$ as follows :

$$
\frac{\partial y^{\prime}}{\partial t}=\frac{\partial}{\partial \theta_{L}}\left[D\left(\frac{\partial \theta_{L}}{\partial x^{\prime}}\right)^{2}\left(\frac{\partial y^{\prime}}{\partial \theta_{L}}\right)\right]+\frac{\partial K}{\partial \theta_{L}}
$$

In similar manner, dependent variables can be derived as following:

$$
\frac{\partial z^{\prime}}{\partial t}=\frac{\partial}{\partial \theta_{L}}\left[D \frac{\partial \theta_{L}}{\partial x^{\prime}}\left(\frac{\partial z^{\prime}}{\partial x^{\prime}}-\left(\frac{\partial y^{\prime}}{\partial x^{\prime}}\right)^{2}\right)\right]
$$

From (2) and (6) to (11), it is evident that: (i) $x$ 'represents horizontal flow and $x$ represents vertical flow; and (ii) $y$ is the result of taking $x-x^{\prime}$, which is due to deviation (or error) of horizontal flow from vertical flow. By including the error $y$ value into horizontal flow equation estimation, it would be practically equating horizontal flow to vertical flow. It is achieved by solving (10) for $y^{\prime}$, instead of (9) for $y$, because in horizontal flow solution for $x$ ' has been solved through $\lambda$ versus $\theta_{L}$ relation. Hence, solution on $y^{\prime}$ would be built on solution on $x$ from horizontal flow. Then, the difference given by $y-y^{\prime}$ would be error $z$, and it would be approximated by $z^{\prime}$, and so forth. When compare to horizontal flow of (5), in a similar manner, vertical flow equation can be represented by (12), where on the left hand side $x$ represent solution to vertical flow equation. On the right hand side: the first term, $\lambda\left(\theta_{L}\right) t^{\frac{1}{2}}$, is the solution to horizontal flow equation only; the second term, $\chi\left(\theta_{L}\right) t^{\frac{2}{2}}$, is the error 
given by $y^{\prime}$; and the third term, $\psi\left(\theta_{L}\right) t^{\frac{3}{2}}$, is the error given by $z^{\prime}$, and so forth.

$$
\begin{aligned}
x= & \lambda\left(\theta_{L}\right) t^{\frac{1}{2}}+\chi\left(\theta_{L}\right) t^{\frac{2}{2}}+\psi\left(\theta_{L}\right) t^{\frac{3}{2}} \\
& +\omega\left(\theta_{L}\right) t^{\frac{4}{2}}+\xi\left(\theta_{L}\right) t^{\frac{5}{2}}+\gamma\left(\theta_{L}\right) t^{\frac{6}{2}} \ldots
\end{aligned}
$$

While in horizontal flow equation (6) is solved by $\lambda\left(\theta_{L}\right) t^{\frac{1}{2}}$, i.e. Boltzmann transformation, the errors $y^{\prime}(10)$ and $z$ are respectively solved by $\chi\left(\theta_{L}\right) t^{\frac{2}{2}}$ and $\psi\left(\theta_{L}\right) t^{\frac{3}{2}}$, i.e. extended Boltzmann transformation, and so forth. The corresponding solutions to (10) and (11) could be generally written as follows:

$\int_{\theta_{n}}^{\theta} f d \theta_{L}=\alpha \frac{d f}{d \theta_{L}}-\beta$

where $f$ represents $\chi, \psi$ and so forth. The $\alpha$ and $\beta$ are known functions of $\theta_{L}$, and these variables varies for different errors under consideration, i.e. $y^{\prime}, z^{\prime}, w^{\prime}$, and so forth. Five pairs of $\alpha$ and $\beta$ are derived corresponding to $\chi, \psi, \omega, \xi$, and $\gamma$, refer to Table I. For instance, $\alpha$ and $\beta$ functions for $y^{\prime}$ is given by:

$\alpha=D\left(\frac{\partial \theta_{L}}{\partial \lambda}\right)^{2} \beta=K_{n}-K$

Similar to solution used to solve horizontal flow equation, two formulae to represent area under the $f-\theta_{L}$ curve by removing the successive rectangles are developed separately and then combined to form a single formula that can be used to govern successive reduction of area under the $f-\theta_{L}$ curve by successive reduction of rectangles. The sequence of formulae and other mathematical manipulation are used to compute $f_{1}, f_{2}, f_{3}, \ldots$. Hence, it generates curve of $f$ versus $\theta_{L}$, e.g.,$\chi$ versus $\theta_{L}, \psi$ versus $\theta_{L}$ and so forth. By specifying the time of infiltration and the volumetric water content values corresponding to the rectangles of the histogram, the spatial variable $(\mathrm{x})$ for vertical flow equation can be calculated by (12).
TABLE I

THE VARIABLES OF $\alpha$ AND $\beta$ ARE DERIVED FOR $\chi, \psi$,

$$
\omega, \xi \text { AND } \gamma
$$

\begin{tabular}{lll}
\hline \hline & $\alpha$ & $\beta$ \\
\hline & $D\left(\frac{d \theta_{L}}{d \lambda}\right)^{2}$ & $K_{n}-K$ \\
$\psi$ & $\frac{2}{3} D\left(\frac{d \theta_{L}}{d \lambda}\right)^{2}$ & $\frac{2}{3} D \frac{d \theta_{L}}{d \lambda}\left(\frac{d \chi}{d \lambda}\right)^{2}$ \\
$\omega$ & $\frac{1}{2} D\left(\frac{d \theta_{L}}{d \lambda}\right)^{2}$ & $\frac{1}{2} D \frac{d \theta_{L}}{d \lambda} \frac{d \chi}{d \lambda}\left[2 \frac{d \psi}{d \lambda}-\left(\frac{d \chi}{d \lambda}\right)^{2}\right]$ \\
$\xi$ & $\frac{2}{5} D\left(\frac{d \theta_{L}}{d \lambda}\right)^{2}$ & $\frac{2}{5} D \frac{d \theta_{L}}{d \lambda}\left(\frac{d \chi}{d \lambda}\right)^{2}\left[2 \frac{d \omega}{d \chi}-3 \frac{d \psi}{d \lambda}+\left(\frac{d \psi}{d \chi}\right)^{2}+\left(\frac{d \chi}{d \lambda}\right)^{2}\right]$ \\
$\gamma$ & $\frac{1}{3} D\left(\frac{d \theta_{L}}{d \lambda}\right)^{2}$ & $\frac{1}{3} D \frac{d \theta_{L}}{d \lambda}\left(\frac{d \chi}{d \lambda}\right)^{2}\left[2 \frac{d \xi}{d \chi}+2 \frac{d \omega}{d \chi} \frac{d \psi}{d \chi}-3 \frac{d \omega}{d \lambda}-3 \frac{d \lambda}{d \chi}\left(\frac{d \psi}{d \lambda}\right)^{2}+4 \frac{d \psi}{d \lambda}\left(\frac{d \chi}{d \lambda}\right)-\left(\frac{d \chi}{d \lambda}\right)^{3}\right]$ \\
\hline \hline
\end{tabular}

\section{RESULT S AND DISCUSSION}

The infiltration medium is sandy loam that has parameters of characteristic functions of van Genuchten [3] as tabulated in Table II. The water infiltration solved using Philip's semianalytical solution with 100 rectangles. The Philip's semianalytical solution was based on step solution outline by Kirkham and Powers [6]. At time equal zero, the volumetric water content is $0.15 \mathrm{~m} 3 \cdot \mathrm{m}-3$. At the surface of infiltration, when time greater than zero, the surface boundary is imposed by near saturation volumetric water content, i.e. $0.4098 \mathrm{~m} 3 \cdot \mathrm{m}-3$. Similarly, a numerical simulation on (2) is implemented for comparis on to the Philip's semi-analytical solution. The results of water infiltration after 6, 12 and 30 minutes are illustrated in Fig. 1. The results indicate Philip's semi-analytical solution has comparable water infiltration data as the numerical solution at different infiltration depths. The governing equation and numerical method is based on finite-difference. The data for water infiltration at different times, i.e. 6, 12, 30 minutes, are tabulated in Table III. The water infiltration data using van Genuchten constitutive functions for matric pressure head and hydraulic conductivity would be useful validation data for other modelers

T ABLE II

SANDY LOAM FITTED By VAN GENUCHTEN CONSTITUTIVE FunCtions AND ITS PARAMETER VALUES [8]

\begin{tabular}{ll}
\hline \hline Parameters & Values \\
\hline$\theta_{s}\left(\mathrm{~m}^{3} \cdot \mathrm{m}^{-3}\right)$ & 0.41 \\
$\theta_{r}\left(\mathrm{~m}^{3} \cdot \mathrm{m}^{-3}\right)$ & 0.065 \\
$\alpha\left(\mathrm{cm}^{-1}\right)$ & 0.075 \\
$K_{s}\left(\mathrm{~cm} \cdot \mathrm{min}^{-1}\right)$ & $7.36806 \times 10^{-2}$ \\
$n$ & 1.89 \\
\hline \hline
\end{tabular}

In soil physics, water flow model is based on Richards' equation. It has been extended by other researchers to model water flow in temperature gradient environment [7]. Also, the water vapor and energy transfers are modeled simultaneously with water flow. Therefore, the ability to validate water flow 
model is necessary before adding multiphase flow and energy transfer equation; because it would help to ease model inspection in case of simulation error.

TABLE II

W ATER INFIL TRATION DATA FROM PHILIP'S SEMI-ANALYTICAL SOLUTION AND VAN GENUCHTEN CONSTITUTIVE FUNCTION ON SANDY LOAM SOIL. WATER INFIL TRATION T IMES ARE 6, 12 AND 30 MINUTES

\begin{tabular}{llll}
\hline$\theta_{L}\left(\mathrm{~m}^{3} \cdot \mathrm{m}^{-3}\right)$ & $\mathrm{t}=6 \mathrm{mins}$ & $\mathrm{t}=12 \mathrm{mins}$ & $\mathrm{t}=30 \mathrm{mins}$ \\
\hline 0.3994 & 2.9315 & 4.7377 & 9.6365 \\
0.3864 & 3.6475 & 5.7333 & 11.0423 \\
0.3734 & 4.0413 & 6.2641 & 11.7769 \\
0.3604 & 4.3063 & 6.6159 & 12.2590 \\
0.3474 & 4.5018 & 6.8729 & 12.6074 \\
0.3344 & 4.6540 & 7.0717 & 12.8741 \\
0.3214 & 4.7768 & 7.2312 & 13.0865 \\
0.3084 & 4.8784 & 7.3627 & 13.2604 \\
0.2954 & 4.9641 & 7.4733 & 13.4061 \\
0.2824 & 5.0375 & 7.5679 & 13.5301 \\
0.2694 & 5.1012 & 7.6498 & 13.6373 \\
0.2564 & 5.1570 & 7.7217 & 13.7310 \\
0.2434 & 5.2066 & 7.7853 & 13.8141 \\
0.2304 & 5.2511 & 7.8425 & 13.8885 \\
0.2174 & 5.2916 & 7.8945 & 13.9562 \\
0.2044 & 5.3293 & 7.9428 & 14.0191 \\
0.1914 & 5.3654 & 7.9891 & 14.0794 \\
0.1784 & 5.4019 & 8.0361 & 14.1406 \\
0.1654 & 5.4441 & 8.0903 & 14.2113 \\
0.1524 & 5.5260 & 8.1957 & 14.3489 \\
\hline
\end{tabular}

Note: the second to fourth columns datasets are in centimeter unit, which is referring to the depth with respect to the volumetric water content in the first column

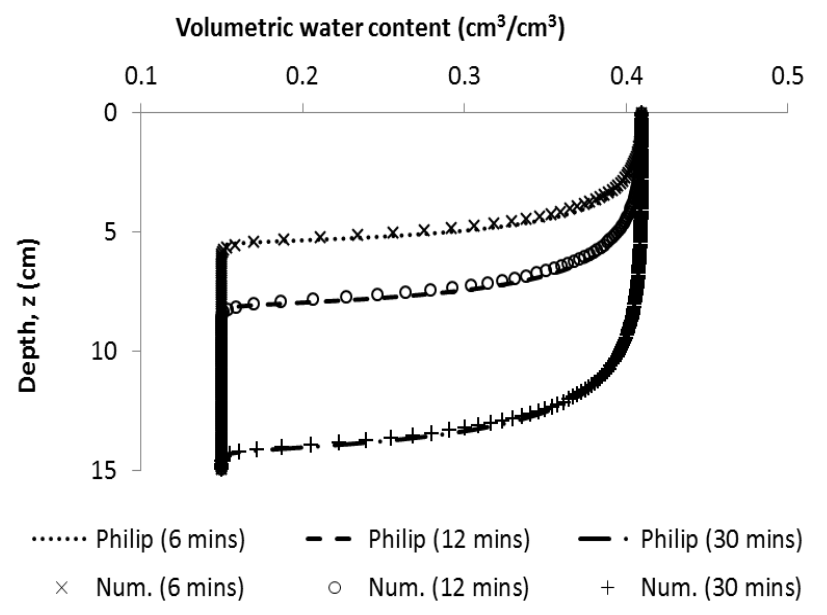

Fig. 1 The comparison of water infiltration in sandy loam soil estimated by Philip's semi-analytical solution and numerical simulation at 6,12 and 30 minutes. Note: Philip is referred to Philip's semi-analy tical solution and Num. is referred to numerical simulation with the spatial size of $0.1 \mathrm{~cm}$

\section{CONCLUSION}

Philip's semi-analytical solution originated from Kirkham and Powers [6] was programmed to model water infiltration into unsaturated soil. A new dataset based on van Genuchten equation was generated for sandy loam. It would be a useful reference for model validation because van Genuchten equation is widely used in soil physics modeling on the importance of the work or suggest applications and extensions.

\section{REFERENCES}

[1] J. Philip, "Numerical Solution of Equations of the Diffusion Type with Diffusivity Concentration-Dependent. II," Australian Journal of Physics vol. 10(1), pp. 29-42, 1957. https://doi.org/10.1071/PH570029

[2] L. A. Richards, "Capillary Conduction of Liquids through Porous Mediums," Journal of Applied Physics, vol. 1(5), pp. 318-333, 1931. doi: $10.1063 / 1.1745010$ https://doi.org/10.1063/1.1745010

[3] M. T. van Genuchten, "A Closed-form Equation for Predicting the Hydraulic Conductivity of Unsaturated Soils," Soil Science Society of America Journal, vol. 44(5), pp. 892-898, 1980. https://doi.org/10.2136/sssaj1980.03615995004400050002x

[4] R. Haverkamp, M. Vauclin, J. Touma, P. J. Wierenga, and G. Vachaud, "A Comparison of Numerical Simulation Models For One-Dimensional Infiltration," Soil Science Society of America Journal, vol. 41(2), pp. 285-294, 1977. https://doi.org/10.2136/sssaj1977.03615995004100020024x

[5] K. Noborio, K. J. McInnes, and J. L. Heilman, "Two-Dimensional Model for Water, Heat, and Solute Transport in Furrow-Irrigated Soil: II. Field Evaluation," Soil Science Society of America Journal, vol. 60(4), pp. 1010-1021, 1996. https://doi.org/10.2136/sssaj1996.03615995006000040008x

[6] D. Kirkham, and W. L. Powers, Advanced soil physics. Wiley, 1972.

[7] J. L. Heitman, R. Horton, T. Ren, I. N. Nassar, and D. D. Davis, "A Test of Coupled Soil Heat and Water Transfer Prediction under Transient Boundary Temperatures," Soil Science Society of America Journal, vol. 72(5), pp. 1197-1207, 2008. https://doi.org/10.2136/sssaj2007.0234

[8] K. Noborio, E. G. Goh, and Y. Ito, "Prediction of Moisture Movement in Soil," Agricultural Environmental Engineering Conference, Japan, 2015. 